\title{
Editorial
}

\section{Local anaesthesia - not a minor anaesthesia}

$\mathrm{f}$ the surgery is planned in local anaesthesia (LA), it does not make it any less serious than it ought to be. The fact is quite the contrary, the patient will be able to peek into the mystique of the world of surgery, and if we do not match his/her expectations and fantasy, he/she may be disillusioned and shattered. To top it, if we fail to perform a truly painless procedure, or provide an ideal operating room environment, we are sure to have a truly dissatisfied patient, and an offending litigation may not be very far behind.

Surgery should be a pleasurable experience and Plastic Surgery in particular should live up to its lofty standards. Whether reconstructive or aesthetic, while the procedure is being performed, the patient should be totally relaxed and painless, and there can be no excuses if he/she is not.

The advantages of LA are several, most of which are well known

- Rapid onset, rapid recovery and early discharge

- Obvious financial benefits

- Relatively high-risk cases can be handled

- In situations where patient co-operation is needed, like in ptosis surgery or tendon surgery, better surgical judgement is possible

The reasons of failing to achieve this status while operating in LA are:

- Poor technique,

- Anatomical variations of sensory and motor nerves,

- Local infection,

- Hypersensitivity due to fear and

- Some forms of Ehlers-Danlos syndrome (EDS).

Poor technique can be because of either improper placement of the local anaesthetic or not waiting long enough for the local anaesthetic to work, or giving the anaesthetic drug too

\begin{tabular}{|l|l|}
\hline \multicolumn{2}{|c|}{ Access this article online } \\
\hline Quick Response Code: & Website: \\
\hline & www.ijps.org \\
\cline { 2 - 2 } & DoI: \\
\hline
\end{tabular}

fast or improper choice of anaesthetic agent, or not giving in adequate amount. The use of topical anaesthetic cream or spray can take away the pain of even a needle prick, and in children, in particular, this may be the magic wand!

Anatomical variations of the target nerve can be a problem. Thus, the standard brachial block may not work as expected and we must be prepared for alternative nerve blocks. A raging localised infection can lessen the effectiveness of local anaesthetic. Local anaesthetics are very $\mathrm{pH}$ sensitive, and with infection, the $\mathrm{pH}$ drops and the environment becomes acidic. In an acid environment, the nerve fibres look to the anaesthesia molecules like they are coated with wax, and thus diffusion into the fibres is very slow.

Highly stressed or anxious patients are poorer candidates for surgery in LA as the anaesthetic may not work as well as when one is relaxed. Adrenaline secreted in response to fear or pain can prevent local anaesthetic from working properly in some people - the onset may be delayed, anaesthesia not pronounced enough, or it may wear off too quickly. The "Gate Control Theory" (Melzack and Wall, 1965) proposes that the body cannot produce a stress response and a relaxation response simultaneously. According to the theory, as a pain impulse is generated, it goes through the spinal cord to the brain where it is interpreted as painful or not painful. The spinal cord is the "gate." The gate sends the signal to the brain, and simultaneously a signal is sent from the brain to the gate to either open or close the gate. If someone is expecting pain, their brain signals the gate to be wide open, and they will then react to the smallest pain impulse. Reassurance from the surgeon and his/her assistance goes a long way to close this gate! Sedation, by lowering anxiety levels, gives the local a much better chance of working.

EDS is a group of rare genetic disorders which affect the connective tissues and present with joint hypermobility, easy bruising and stretchy skin. These patients are poor candidates for surgery in LA.

Of the five senses, hearing, smell, sight, touch and taste, only the last one is of little concern when we are operating 
in LA. The first four are essential and LA is only knocking off the "touch." The patient is wide awake and alert and so should be all the medical professionals in the operating room. Unfortunately, the word "alert" is often changed to the overused word "vigilant" and after being declared very important, is then, in practice, disregarded. During surgery, listening to the doctor/nurse conversations can be very disturbing. Outside the operating room, they view these professionals as exactly that and their conversations are always very proper. While under the knife if they hear about the bad night partying the professionals had, chatter about all types of things not associated with what they are doing or even "flirt" with each other, it gets a bit scary for the patient. They have every right to think that the professionals are not concentrating on the surgery and they are not considering the patient their first priority. To the patient, operating room chatter means the professionals are not thinking about the patient. The conversations which doctors and nurses may find innocuous and routine banter are, to the patient, quite funny sometimes, but unsettling most of the time. The patient should always feel he/she is not "just a patient" but "your patient" and so "your responsibility."

It is vital to observe the general operating room surroundings and reduce unnecessary noise. Excessive operating room background noise from music or too many people talking at once are distractions best avoided. Noises may distract you from hearing important things going wrong with your patient or that some equipment is malfunctioning.

- An oxygen concentrator may be making a noise.

- The patient's breathing may have become noisy, or changed in frequency; possibly there is airway obstruction or inadequate anaesthesia.

- The suction or diathermy may be making an unusual sound, perhaps indicating a leak or disconnection.

A patient in LA has an intact sense of hearing, smell and sight. An offensive smell from a dirty suction machine, operating table or mattress, leaking anaesthetic agent, overheating motor or electric plug and blocked operating room drains can never be a pleasant experience. Similarly, the sight of someone falling asleep in the operating room, eating sandwich, watching movie on laptop, reading newspaper, discussing roster, cracking jokes are things which simply have no place in the operating room, whether the patient is fast asleep or wide awake. This only reflects the fact that the professionals are not proud of what they do and so do not take their own work seriously.

The other major issue is the "memory effect." Some patients have vivid memories of the entire procedure, and even if the surgery was smooth and uncomplicated, have noxious memories of the whole event. Judicious use of intravenous adjuvants may help prevent this.

It is a good idea to check whether the temperature of the room is too hot for the staff or too cold for the patient and assess whether a warming blanket is needed. Warming of injectable solutions, use of sodium bicarbonate to reduce the sting of the acid solution, using an appropriate bore needle, and constant reassurance are all small but significant measures to make the task easier and less painful. Children particularly need to be handled with tender love and care and it is good idea to assign a nurse to become a parttime mother to the child exclusively for this purpose. It is a fundamental rule in any anaesthesia that you must never leave your patient unattended and LA is no exception. As a matter of fact, it is essential to have complete monitoring systems in place and a working knowledge of the drugs involved. Safe dosages must be kept in mind as well as the possibility of accidental intravascular injections. Constant aspiration, while useful, is not of much value when very small bore needles are used. In such cases, careful observation of the hub of the needle will warn the surgeon about intravascular position of the needle.

Good surgical practice continues even after the procedure is completed. During transportation, a numb limb should not get injured or placed in a position of stress, and it is a good habit to thank the nurses and assistants at the end of the surgery for the excellent service they had just rendered. The patient is impressed and almost always expresses his/her gratitude to everybody immediately thereafter.

Surgery may be routine for you, but for a patient it is a once in a lifetime experience! Let them enjoy it and cherish it for the rest of their lives. Remember, they are our most potent advertising billboards and most effective calling cards!

\section{Surajit Bhattacharya}

Editor, IJPS

E-mail: surajitbh@yahoo.co.in

How to cite this article: Bhattacharya S. Local anaesthesia - not a minor anaesthesia. Indian J Plast Surg 2011;44:375-6. 